\title{
Low-field magnetic resonance anatomic features of the neonatal foal thorax: an ex vivo study
}

\author{
Alberto ARENCIBIA ${ }^{1{ }_{\star}}$, José Ángel SANDOVAL ${ }^{2}$, Francisco $\mathrm{GIL}^{3}$, José Raduán JABER ${ }^{1}$, \\ Diego BLANCO ${ }^{1}$, Andrés DIZ ${ }^{4}$, José María VÁZQUEZ ${ }^{3}$ \\ ${ }^{1}$ Department of Morphology, Faculty of Veterinary, University of Las Palmas de Gran Canaria, Spain \\ ${ }^{2}$ Veterinary Hospital, University of Leon, Spain \\ ${ }^{3}$ Department of Anatomy, Faculty of Veterinary, University of Murcia, Spain \\ ${ }^{4}$ Department of Anatomy, Faculty of Veterinary, University of Córdoba, Spain
}

Received: 22.04 .2015

- $\quad$ Accepted/Published Online: 11.06 .2015

Printed: 28.08 .2015

\begin{abstract}
The purpose of this study was to describe the normal appearance of the bony and soft tissue structures of the thorax of neonatal foals by low-field magnetic resonance imaging (MRI) and the use of anatomical dissections and gross sections. Three purebred Spanish neonatal foals that died for medical reasons unrelated to thoracic disease were used to obtain T1- and T2-weighted MR images using a magnet of 0.2 Tesla. Anatomic structures on the MR images were evaluated according to characteristics of signal intensity of the different organic tissues. The bones, different associated muscles, diaphragm, trachea, main bronchi, lungs, esophagus, heart chambers, and associated great vessels were well-visualized and evaluated with a low magnetic field. MRI provided correct discrimination of thoracic structures, and this information could be used as an initial anatomic reference for the interpretation of MR images of the thoraxes of neonatal foals.
\end{abstract}

Key words: Neonatal foal, thorax, anatomy, magnetic resonance imaging

\section{Introduction}

In human medicine, computed tomography (CT) and magnetic resonance imaging (MRI) are diagnostic techniques used in assessment of the thorax because of their high spatial and temporal resolutions, large fields of view, and multiplanar imaging reconstruction capabilities (13). Manipulation of the relative signal intensities of normal and abnormal tissue through appropriate use of MRI pulse sequences, the absence of ionizing radiation, and the ability to acquire images in nonaxial imaging planes are the relative advantages of MRI over CT $(4,5)$. The evaluation of anatomic structures of the thorax is laborious due to its complex organization, which makes it difficult to diagnose morphologic alterations by means of physical examination and conventional radiographic studies (6). MRI may be a good alternative to conventional radiology. MRI provides views of the structures without superimposition, a capacity to discriminate the different tissues $(7,8)$, and high diagnostic value (9). MRI also provides a larger field of view and depicts the complete chest including abnormalities of the surrounding mediastinum and inflammatory processes (1). Therefore, MRI has been used to assess chest wall, mediastinum, and diaphragm diseases $(10,11)$, as well as the neonatal heart (12-14). In addition, other experimental studies on animal models have demonstrated the utility of MRI for evaluation of thoracic structures $(15,16)$.

In equine medicine, radiology, ultrasound, and echocardiography are the main imaging modalities used for diagnosis of thoracic disorders in neonatal foals (17-19). MRI has also been used in these animals, but information was limited to a few clinical reports on the head (20) and musculoskeletal system (21). Specific thoracic pathologies in foals such as pneumonia, atelectasis, edema, hyperemia, emphysema, cavities of the pericardium, and dilatation of the heart have also been described by MRI (7); however, to date, a detailed anatomical study in thorax of neonatal foals using MRI has not been done. Therefore, the aim of this study was to describe the normal anatomy of the neonatal foal thorax using parasagittal, transverse, and dorsal MR images, as well as anatomical dissections and gross sections.

\section{Materials and methods}

Two female and one male purebred Spanish foals that died for reasons unrelated to thoracic disorder were used.

\footnotetext{
*Correspondence: alberto.arencibia@ulpgc.es
} 
Animals were 1-2 days old with weights between 50 and $55 \mathrm{~kg}$. MR imaging was performed within $24 \mathrm{~h}$ of death, to minimize postmortem changes, at the Veterinary Hospital of Leon University (Spain). An open-bore magnet configuration operating at a field strength of 0.2 Tesla (Signa Profile Excite, GE Medical Systems, Spain) was used. Dimensions of the bore were $120 \times 44 \mathrm{~cm}$. Each animal was positioned in ventral recumbency for the imaging procedure. An RF head coil with dimensions of $54 \times 42 \mathrm{~cm}$ was used for image acquisition. Fast spin-echo pulse sequences were used to obtain T1-weighted and T2weighted images in parasagittal and transverse planes, as well as T1-weighted images in the dorsal plane. MRI parameters used in this study are presented in the Table.

Anatomical dissections were performed in two foals to aid in identification of parasagittal MR images. The only postmortem changes consisted of gravitational pulmonary hypostasis (livor mortis). The third foal was frozen and then sectioned transversely ( $2 \mathrm{~cm}$ thick) using an electric bandsaw to correspond with transverse MR images. The thoracic structures studied in MR images were evaluated according to the characteristic signal intensity of different tissues and correlated with structures identified in the corresponding anatomical dissections and transverse gross sections. The nomenclature used for designating anatomical structures of the thorax was in accordance with official anatomical terminology (22).

\section{Results}

The results are shown in seven representative figures. Figures 1 and 2 are parasagittal MR images and the corresponding anatomic dissections. Figure 1 was obtained
$0.95 \mathrm{~cm}$ lateral to the spine on the right, and Figure 2 was obtained $0.95 \mathrm{~cm}$ lateral to the spine on the left. Transverse MR images (Figures 3-6) are presented in cranial to caudal progression from the level of the of brachiocephalic trunk and cranial cava vein to the caudal cava vein in the right atrium. These images are displayed so that the right side of the foal is to the viewer's left and the dorsal is at the top. Figure 7 is a composition of three images; one left parasagittal MR image, where the lines represent the approximate locations of two dorsal MR images displayed from the level of the tracheal carina (Figure 7A) to the pulmonary trunk (Figure 7B).

Correlation of anatomic structures was performed between the MR images, anatomical dissections, and gross sections. The bony structures were defined on the MR images. Thus, the thoracic vertebrae (with the vertebral body; vertebral arch; and the corresponding articular, transverse, and spinous processes), the ribs (including the costal bone and costal cartilage), sternum, and scapula could be identified. Intervertebral discs, as well as the costovertebral, costochondral, and sternocostal joints were also visible. In addition, muscles associated with the thorax (epiaxial and thoracic wall muscles) and other thoracic structures such as pericardial and mediastinal fat were identified. Air-filled structures such as the trachea, main bronchi, parenchyma of the right and left lungs, and esophagus were easily visualized in MR images and the anatomical dissections and gross sections.

The heart chambers and associated blood vessels were well displayed in the MR images and the corresponding anatomical preparations. The cranial and caudal vena cava, leading into the right atrium, and the right azygos, ending

Table. Parameters used for the MRI study.

\begin{tabular}{|c|c|c|c|c|c|}
\hline Parameters & $\begin{array}{l}\text { Sagittal } \\
\text { T1W }\end{array}$ & $\begin{array}{l}\text { Sagittal } \\
\mathrm{T} 2 \mathrm{~W}\end{array}$ & $\begin{array}{l}\text { Transverse } \\
\text { T1W }\end{array}$ & $\begin{array}{l}\text { Transverse } \\
\text { T2W }\end{array}$ & $\begin{array}{l}\text { Dorsal } \\
\text { T1W }\end{array}$ \\
\hline Repetition time (TR) (ms) & 400 & 3120 & 420 & 2640 & 500 \\
\hline Echo time (TE) (ms) & 19 & 125 & 22 & 131 & 26 \\
\hline Field of view $(\mathrm{cm})$ & $80 \times 37$ & $80 \times 37$ & $80 \times 37$ & $80 \times 37$ & $80 \times 37$ \\
\hline Acquisition matrix & $512 \times 512$ & $512 \times 512$ & $256 \times 256$ & $256 \times 256$ & $256 \times 256$ \\
\hline Slice thickness (mm) & 9 & 9 & 9 & 9 & 9 \\
\hline Interslice spacing (mm) & 9.5 & 9.5 & 9.5 & 9.5 & 9.5 \\
\hline Number of excitations & 2 & 4 & 2 & 3 & 2 \\
\hline Images in acquisition & 11 & 11 & 20 & 20 & 5 \\
\hline Acquisition number & 1 & 1 & 1 & 1 & 1 \\
\hline Total acquisition time (min) & 7 & 7 & 12 & 12 & 5 \\
\hline
\end{tabular}




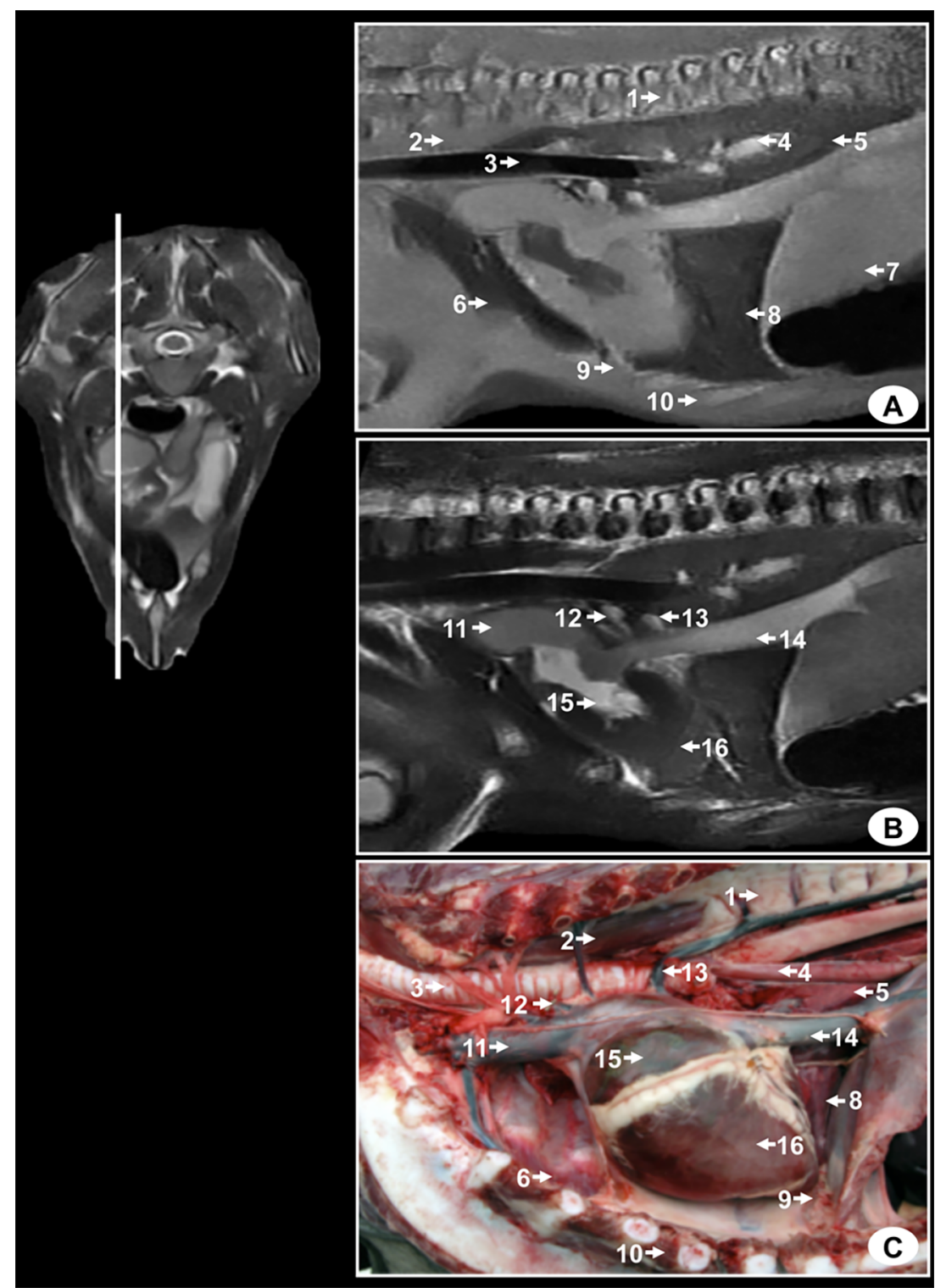

Figure 1. Parasagittal MR images $0.95 \mathrm{~cm}$ right lateral to the spine. (A) T1-weighted; (B) T2-weighted; and the corresponding anatomic dissection (C). Line represents the approximate location for parasagittal MR image. 1 . Body of thoracic vertebra; 2 . longus colli muscle; 3 . trachea; 4. esophagus; 5 . caudal lobe of the left lung; 6 . cranial lobe of the left lung; 7. liver; 8. accessory lobe of the right lung; 9. pericardium, sternopericardial ligament; 10. sternum; 11 . cranial cava vein; 12 . pulmonary veins and right pulmonary artery; 13. right azygos vein; 14. caudal cava vein; 15. right atrium; 16. right ventricle.

in the cranial vena cava, were clearly appreciated (Figure 1). In Figure 2, the course of the ascending aorta and the main branches such as the brachiocephalic trunk and the descending aorta were visible. These vascular structures were more easily appreciated in the anatomical dissection. Furthermore, the pulmonary trunk rising from the right ventricle was clearly visible in the parasagittal MR image and the corresponding anatomical dissection. In addition, the vascular structures of the pulmonary parenchyma such as the lobar pulmonary artery and vein, as well as the lobar bronchus, were well defined on transverse MR images (Figures 3-6). In Figure 7, the main vascular structures were readily identified on the dorsal plane MR images.

In the present study, MRI provided correct detail of the normal neonatal foal thorax and good discrimination between soft and mineralized tissues in T1- and T2- 


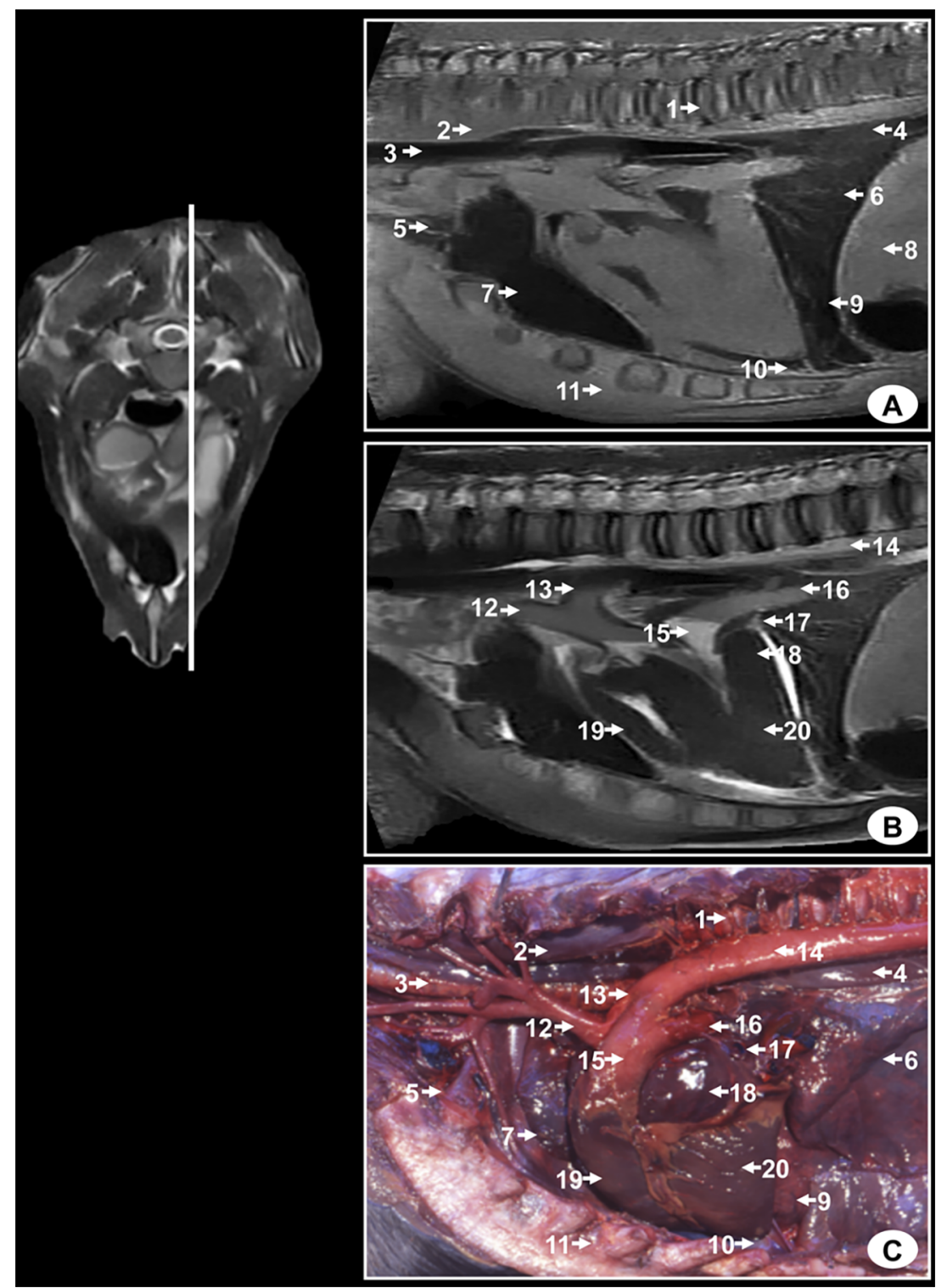

Figure 2. Parasagittal MR images $0.95 \mathrm{~cm}$ left lateral to the spine. (A) T1-weighted; (B) T2-weighted; and the corresponding anatomic dissection $(C)$. Line represents the approximate location for parasagittal MR image. 1. Body of thoracic vertebra; 2 . longus colli muscle; 3 . trachea; 4 . esophagus; 5 . mediastinum, thymus; 6 . caudal lobe of the right lung; 7. cranial lobe of the right lung; 8 . liver; 9. accessory lobe of the right lung; 10. pericardium, sternopericardial ligament; 11. sternum; 12. brachiocephalic trunk; 13. aortic arch; 14. descending aorta; 15. pulmonary trunk; 16. left pulmonary artery; 17. pulmonary veins; 18 . left atrium; 19. right ventricle; 20 . left ventricle.

weighted MR images. Therefore, cortical and subchondral bone was black (signal void), and the bone marrow was gray (intermediate signal intensity) in both T1- and T2weighted MR images. Intervertebral discs, costovertebral, costochondral, and sternocostal joints showed white (high signal intensity) in T1-weighted MR images and gray in T2-weighted MR images. The associated muscles of the thoracic wall appeared with intermediate signal intensity in T1-weighted images and dark gray (low signal intensity) in T2-weighted MR images.

The lumen of the trachea, main bronchi, and esophagus were black (signal void) in both MR images. However, the walls corresponding to tracheal and bronchial cartilage and esophagus muscles appeared with intermediate signal 


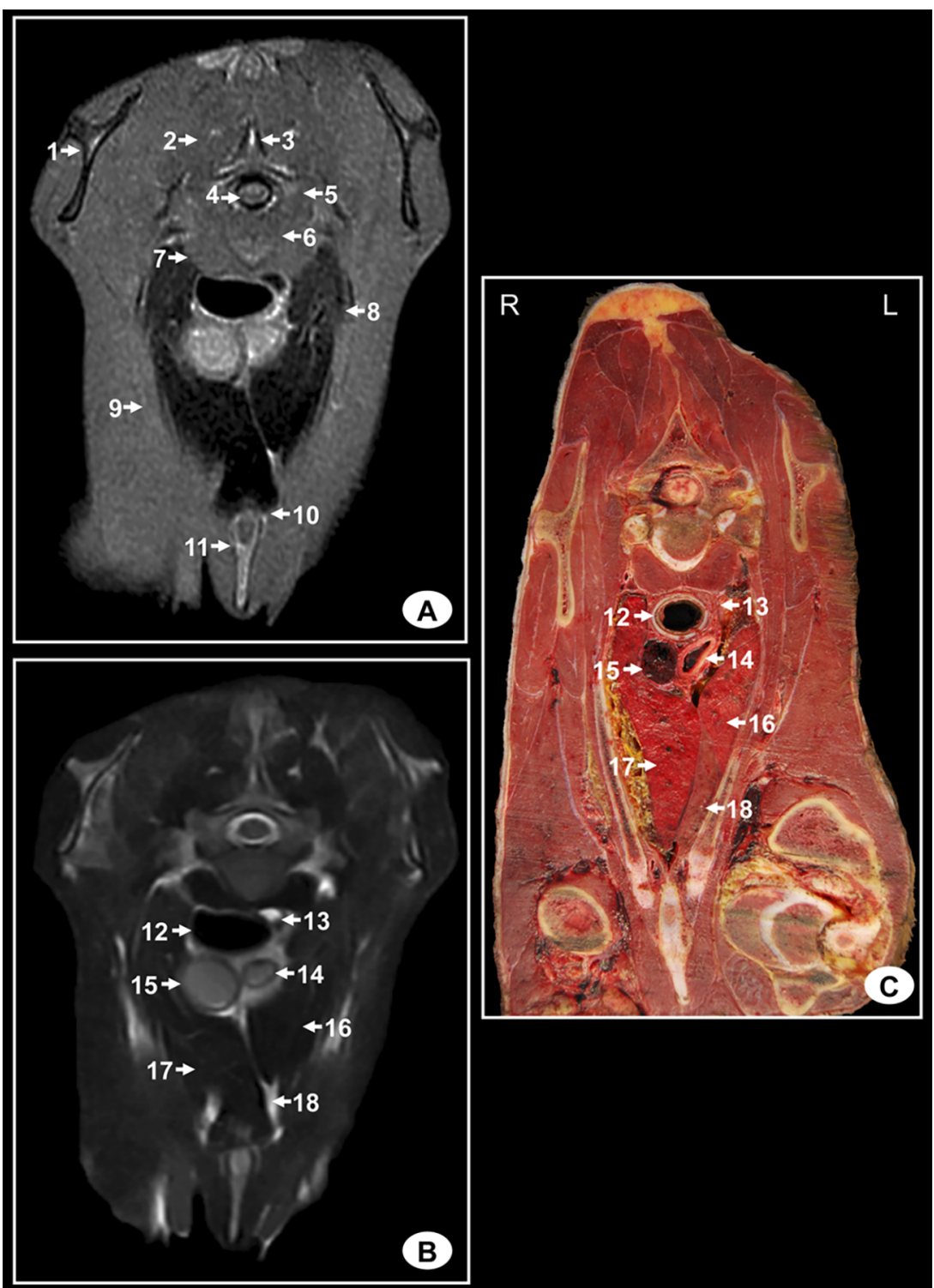

Figure 3. (A) T1-weighted transverse MR image; (B) T2-weighted transverse MR image; and $(C)$ anatomic section at the level of brachiocephalic trunk and cranial cava vein. $\mathrm{R}$ is right, $\mathrm{L}$ is left. 1. Scapula; 2. epiaxial muscles; 3. spinous process of thoracic vertebra; 4. spinal cord; 5 . transverse process of thoracic vertebra; 6 . body of thoracic vertebra; 7. longus colli muscle; 8. costal bone; 9. thoracic wall muscles; 10 . costal cartilage; 11. sternum; 12. trachea; 13. esophagus; 14. brachiocephalic trunk; 15 . cranial cava vein; 16. cranial lobe of the left lung; 17. cranial lobe of the right lung; 18. mediastinum, thymus.

intensity. Moreover, the parenchyma of the right and left lungs showed low signal intensity, but there were some differences in appearance between the pulmonary lobes, with a higher intensity signal in the caudal and accessory lobes, and the cranial lobes.

The lumens of the atrial and ventricular chambers and associated blood vessels appeared uniformly T1W hyperintense and $\mathrm{T} 2 \mathrm{~W}$ hypointense to $\mathrm{T} 2 \mathrm{~W}$ hyperintense.
At the level of the pulmonary parenchyma, the vascular structures had higher signal intensity than the lung.

\section{Discussion}

Several MRI studies have been done on living (15) and cadaveric specimens to evaluate anatomic structures of the thorax $(7,23)$. The primary difference between a live and cadaver animal is the signal intensity from vessels. Cardio- 


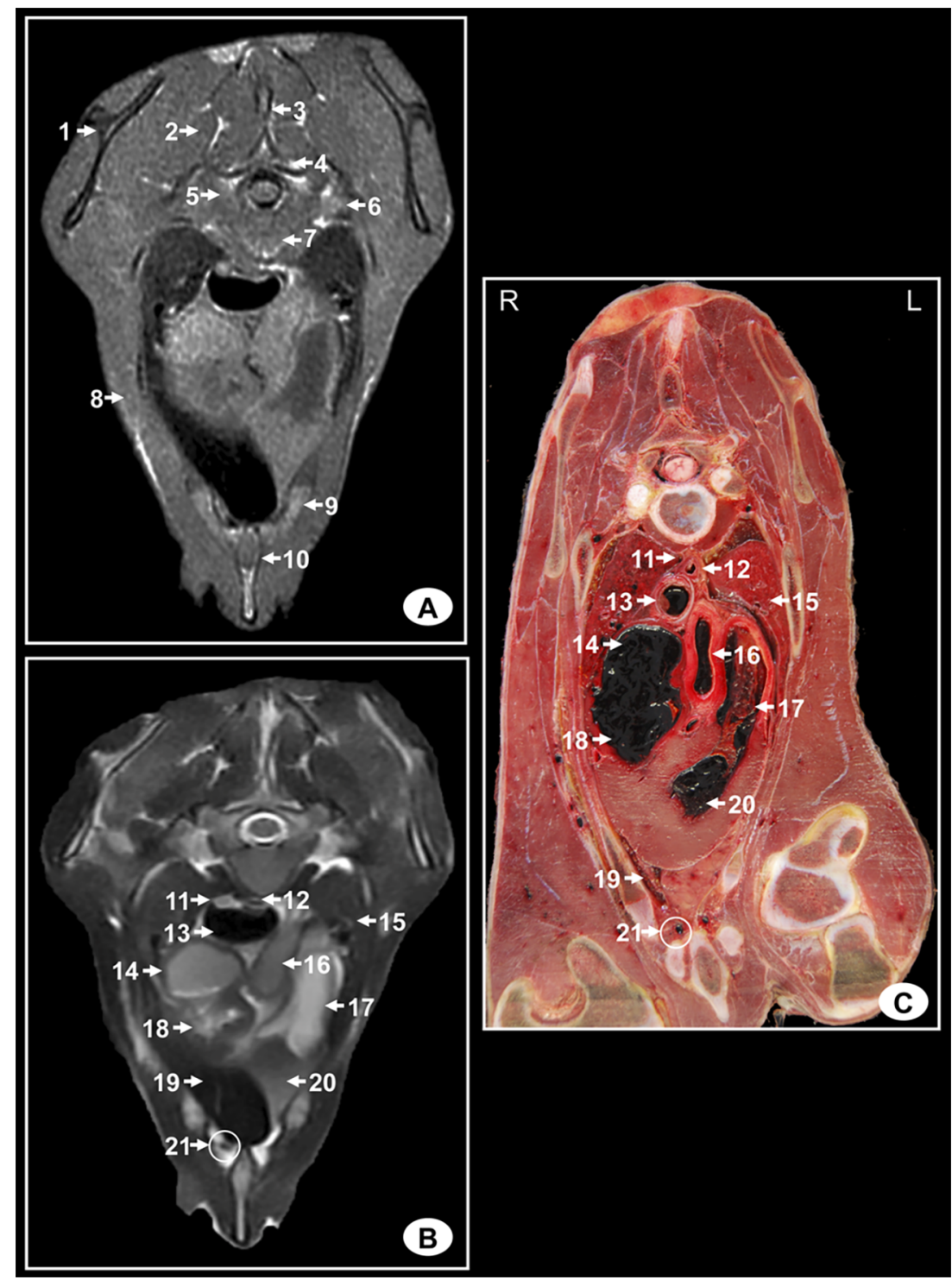

Figure 4. (A) T1-weighted transverse MR image; (B) T2-weighted transverse MR image; and $(\mathrm{C})$ anatomic section at the level at the level of ascending aorta. $\mathrm{R}$ is right, $\mathrm{L}$ is left. 1. Scapula; 2. epiaxial muscles; 3 . spinous process of thoracic vertebra; 4 . articular process of thoracic vertebra; 5 . transverse process of thoracic vertebra; 6 . costal bone; 7 . body of thoracic vertebra; 8 . thoracic wall muscles; 9. costal cartilage; 10. sternum; 11. right azygos vein; 12 . esophagus; 13 . trachea; 14 . cranial cava vein; 15 . caudal lobe of the left lung; 16. ascending aorta; 17. pulmonary trunk; 18. right atrium; 19. caudal lobe of the right lung; 20. right ventricle; 21 . internal thoracic artery and vein.

vascular structures appear T1W hyperintense and $\mathrm{T} 2 \mathrm{~W}$ hypointense. In our study, the relative signal intensity of the lumens of cardiovascular structures was likely due to blood degradation and a predominance of intracellular (T2W hypointense) and extracellular (T2W hyperintense) methemoglobin. In addition, the slight changes in signal intensity of the pulmonary parenchyma could be due to gravitational pulmonary hypostasis, which results in an uneven distribution of blood.
Parasagittal, transverse, and dorsal MR images obtained in this study are useful for the evaluation of the bony and soft tissue structures of the thorax. Parasagittal acquisition is preferred for the evaluation of midline thoracic vascular structures. In the transverse plane, the anatomical relationships of bony structures, thoracic wall muscles, heart, lungs, and associated blood vessels are more easily appreciated, while dorsal MR images provide certain standard references for the size and position of 


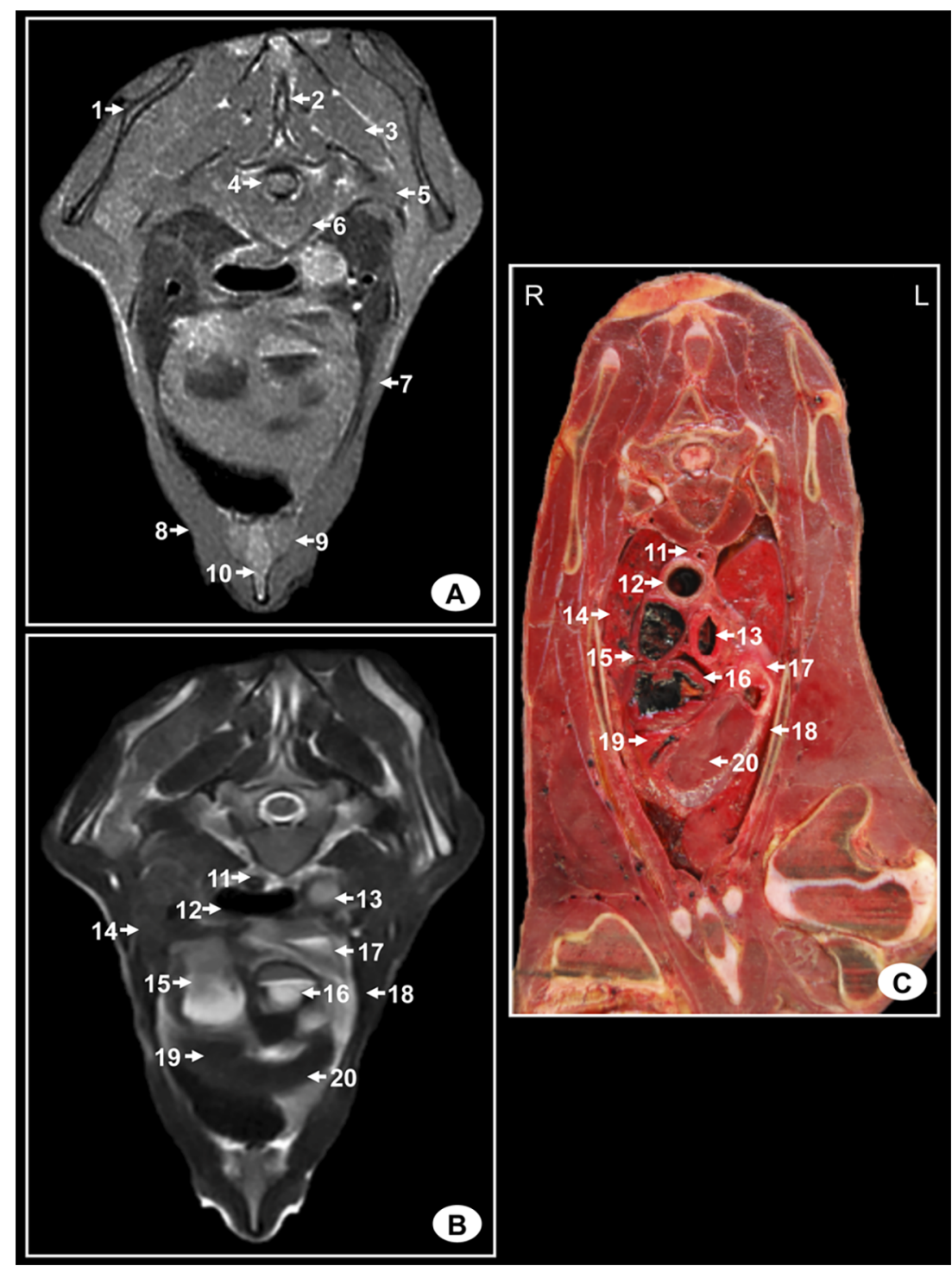

Figure 5. (A) T1-weighted transverse MR image; (B) T2-weighted transverse MR image; and (C) anatomic section at the level of aortic bulb. $\mathrm{R}$ is right, $\mathrm{L}$ is left. 1. Scapula; 2. spinous process of thoracic vertebra; 3 . epiaxial muscles; 4 . spinal cord; 5 . costal bone; 6. body of thoracic vertebra; 7. thoracic wall muscles; 8 . pectoral muscles; 9 . costal cartilage; 10. sternum; 11. esophagus; 12 . trachea; 13 . descending aorta; 14 . caudal lobe of the right lung; 15. right atrium; 16. aortic bulb; 17. left atrium; 18. caudal lobe of the left lung; 19. right ventricle; 20 . left ventricle.

the heart and associated blood vessels. A thick slice and interslice gap were used for all of the sequences to select anatomical levels of clinical interest.

The use of MRI in equine medicine is currently limited because of its expense, availability, and the problems of acquiring MR images in older foals and adult horses due to their physical size. However, for neonatal foals, MRI may be a clinically effective imaging modality $(7,20)$. This study has shown that low-field MRI is useful for the visualization of bone, soft tissues, and fluids of the thoraxes of neonatal foals, as demonstrated in humans (24) and neonatal foals (7).

In this study, FSE T1W and T2W MR images were obtained using a $0.2 \mathrm{~T}$ low-field open-bore configuration MR system. These sequences provided a good depiction of anatomical structures. Some reports describe thoracic diseases using the same sequences $(1,25)$. However, in live animals the technical changes including use of closed-bore 1.5 T high-field configuration, a cardio-respiratory gating, 


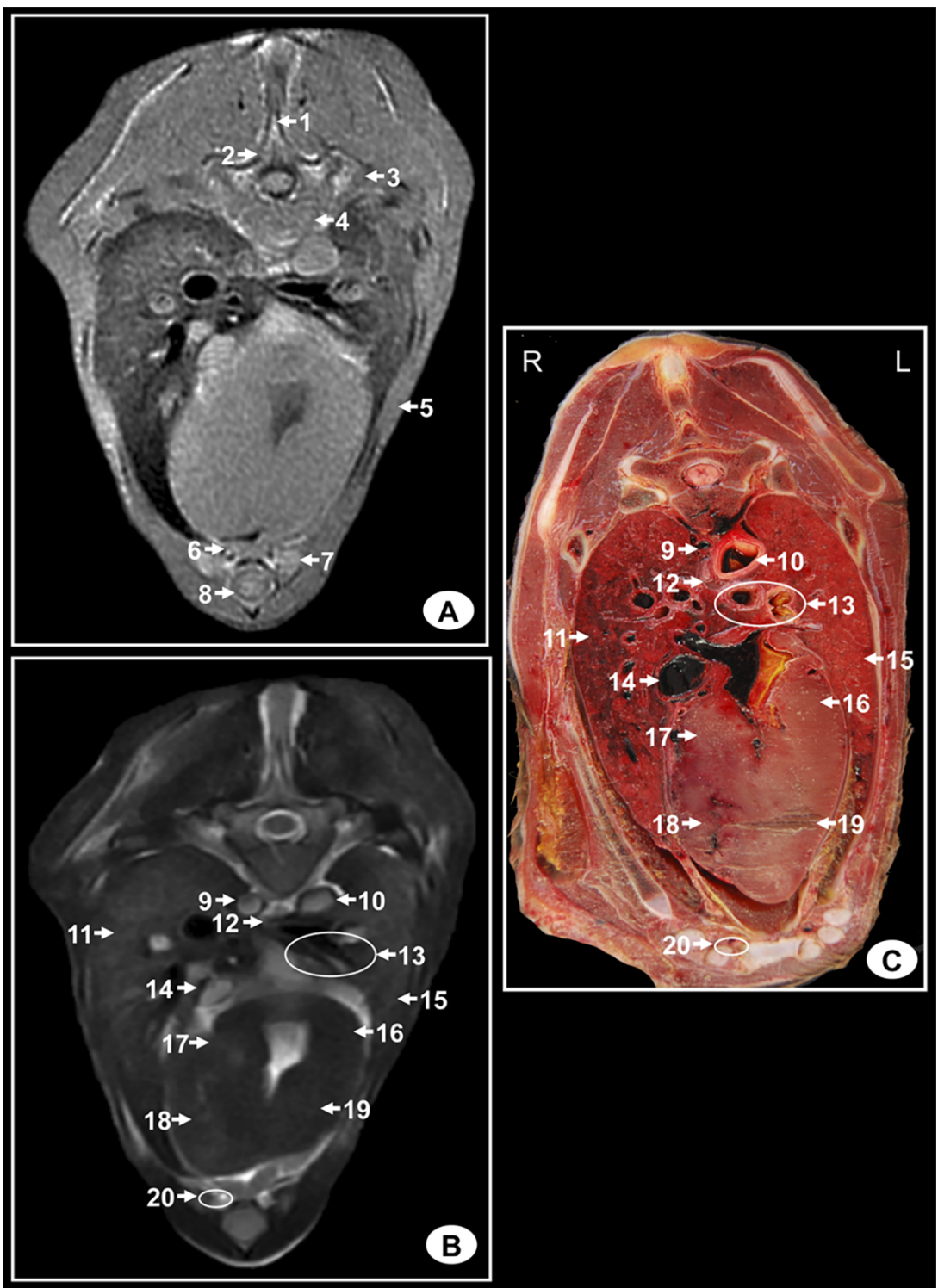

Figure 6. (A) T1-weighted transverse MR image; (B) T2-weighted transverse MR image; and $(\mathrm{C})$ anatomic section at the level of caudal cava vein in right atrium. $\mathrm{R}$ is right, $\mathrm{L}$ is left. 1. Spinous process of thoracic vertebra; 2 . articular process of thoracic vertebra; 3. costal bone; 4 . body of thoracic vertebra; 5 . thoracic wall muscles; 6 . pericardium, sternopericardial ligament; 7. costal cartilage; 8. sternum; 9. right azygos vein; 10. descending aorta; 11 . caudal lobe of the right lung; 12. esophagus; 13 . left lobar pulmonary vein, lobar arterial branch, and the lobar bronchus; 14 . caudal cava vein; 15 . caudal lobe of the left lung; 16. left atrium; 17. right atrium; 18. right ventricle; 19. left ventricle; 20. internal thoracic artery and vein.

and different sequences produce high-quality diagnostic images without artefacts $(4,13)$.

This anatomical study can aid in the diagnosis of different thoracic disorders such as malformations (17), pericardial diseases (1), thoracic wall injuries (11), inflammation (26), infection (27), and masses (25). In the future, it would be interesting to perform further studies with low and higher magnetic fields on live animals, along with the administration of a contrast medium to establish a protocol for magnetic resonance angiography.

In conclusion, gross dissections and anatomical sections are useful tools for the correct morphological and topographic evaluation of MR images of neonatal foal thoraxes. 


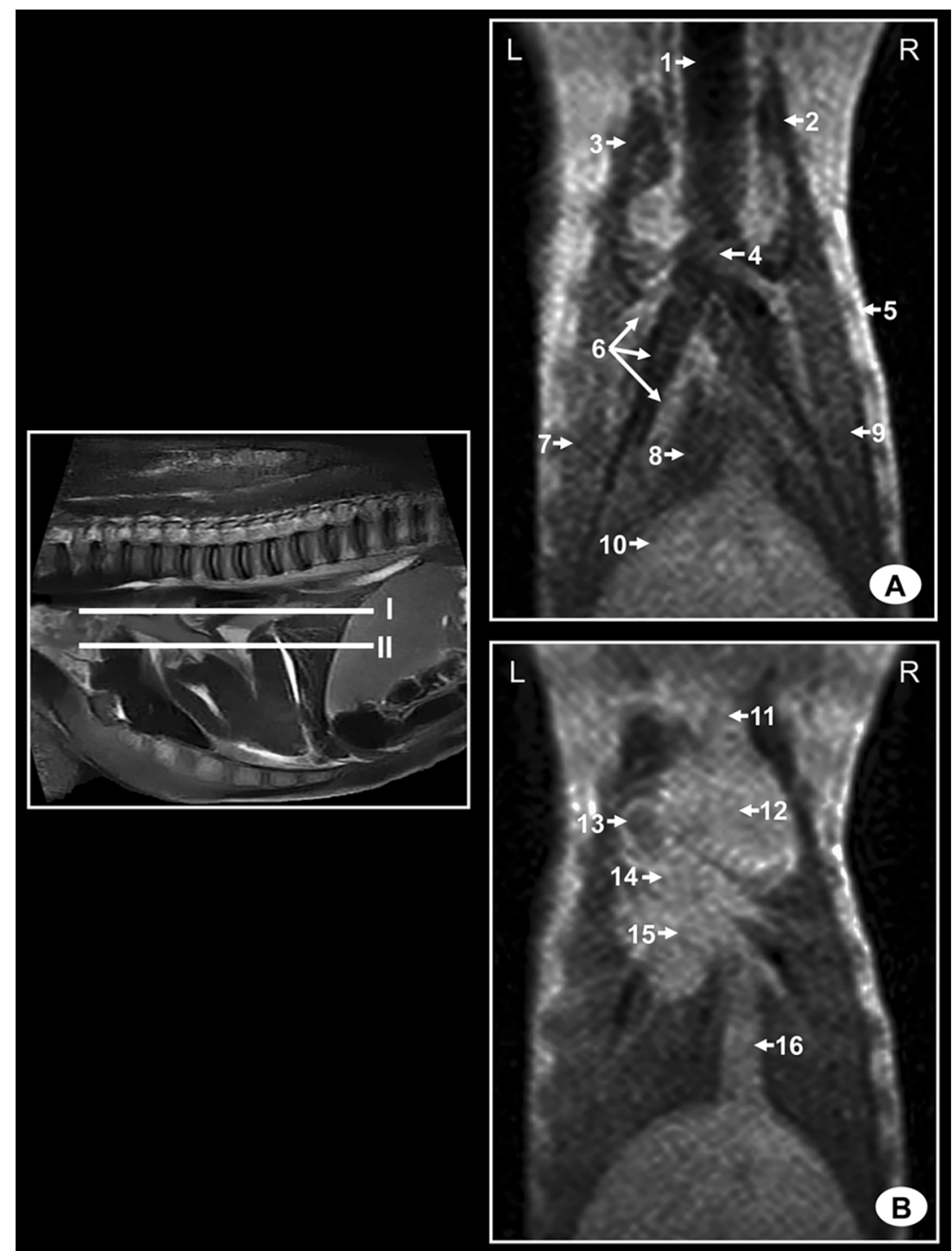

Figure 7. Dorsal MR images of the thorax. Each line and number (I,II) represents the location for each MR image of the following MR images. (A) T1-weighted dorsal MR image at the approximate level of tracheal carina (level I) and (B) T1-weighted dorsal MR image at the approximate level of pulmonary trunk (level II). $\mathrm{R}$ is right, $\mathrm{L}$ is left. 1. Trachea; 2. cranial lobe of the right lung; 3 . cranial lobe of the left lung; 4 . tracheal carina; 5. costal bone and thoracic wall muscles; 6 . left lobar pulmonary vein, lobar arterial branch, and lobar bronchus; 7. caudal lobe of the left lung; 8. accessory lobe of the right lung; 9. caudal lobe of the right lung; 10. liver; 11 . cranial cava vein; 12 . right atrium; 13. pulmonary trunk; 14 . ascending aorta; 15 . left atrium; 16. caudal cava vein.

Findings from this cadaveric study indicated that sagittal and transverse MR images of the thorax obtained with low-field equipment were adequate for anatomic evaluation.

\section{Acknowledgments}

This work was supported by funds from the Department of Morphology of Las Palmas de Gran Canaria University (Spain) and the Department of Anatomy of Murcia University (Spain). 


\section{References}

1. Alter P, Figiel JH, Rupp TP, Bachmann GF, Maisch B, Rominger MB. MR, CT, and PET imaging in pericardial disease. Heart Fail Rev 2013; 18: 289-306.

2. Cuddy LC, Maisenbacher HW, Vigani A, Berry C. Computed tomography angiography of coarctation of the aorta in a dog. J Vet Cardiol 2013; 15: 277-281.

3. Stein E, Mueller GC, Sundaram B. Thoracic aorta (multidetector computer tomography and magnetic resonance evaluation). Radiol Clin North Am 2014; 52: 195-217.

4. Contreras S, Vázquez JM, De Miguel A, Morales M, Gil F, López $\mathrm{O}$, Arencibia A. Magnetic resonance angiography of the normal canine heart and associated blood vessels. Vet J 2008; 178: $130-132$.

5. Rajiah P. CT and MRI in the evaluation of thoracic aortic diseases. Int J Vasc Med 2013; 79: 71-89.

6. Bedenice D, Heuwieser W, Brawer R, Solano M, Rand W, Paradis MR. Clinical and prognostic significance of radiographic pattern, distribution, and severity of thoracic radiographic changes in neonatal foals. J Vet Intern Med 2003; 17: 876-886.

7. Fitz J, Gerhards H. Kernspintomographie im Bereich von Thorax und Abdomen beim Fohlen. Pferdeheilkunde 2005; 21 : 115-123 (article in German with an abstract in English).

8. Venkatesh V, Verdini D, Ghoshhajra B. Normal magnetic resonance imaging of the thorax. Magn Reson Imaging Clin $\mathrm{N}$ Am 2011: 19; 489-506.

9. Wagner M, Böwing B, Kuth R, Deimling M, Rascher W, Rupprecht T. Low field thoracic MRI-a fast and radiation free routine imaging modality in children. Magn Reson Imaging 2001; 19: 975-983.

10. Takahashi K, Al-Janabi NJ. Computed tomography and magnetic resonance imaging of mediastinal tumors. J Magn Reson Imaging 2010; 32: 1325-1339.

11. Subhas N, Kline MJ, Moskal MJ, White LM, Recht MP. MRI evaluation of costal cartilages injuries. Am J Roentgenol 2008; 191: 129-132.

12. Krishnamurthy R, Lee E. Congenital cardiovascular malformations: noninvasive imaging by MRI in neonates. Magn Reson Imaging Clin N Am 2011; 19: 813-822.

13. Dong SZ, Zhu M, Li F. Preliminary experience with cardiovascular magnetic resonance in evaluation of fetal cardiovascular anomalies. J Cardiovasc Magn Reson 2013; 202: 971-981.

14. Ghadimi Mahani M, Lu JC, Rigsby CK, Krishnamurthy R, Dorfman AL, Agarwal PP. MRI of pediatric cardiac masses. Am J Roentgenol 2014: 202; 971-981.
15. Hernandez RJ, Strouse PJ, Londy FJ, Wakefield TW. Gadolinium-enhanced MR angiography (Gd-MRA) on the thoracic vasculature in an animal model using double dose gadolinium and quiet breathing. Pediatr Radiol 2001; 31; 589593.

16. Ren X, Wang W, Zhang X, Pu Y, Wang M, Jiang T. The comparative study of magnetic resonance angiography diagnosis and pathology of blunt vertebral artery injury. Spine 2006; 31: 2124-2129.

17. Reef VB. Cardiovascular disease in the equine neonate. Vet Clin North Am Equine Pract 1985; 1: 117-129.

18. Mazan MR, Vin R, Hoffman AM. Radiographic scoring lacks predictive value in inflammatory airway disease. Equine Vet J 2005; 37: 541-545.

19. Neil KM, Charman RE, Vasey JR. Rib osteomyelitis in three foals. Aust Vet J 2010; 88: 96-100.

20. Chaffin MK, Walker MA, McArthur NH, Perris EE, Matthews NS. Magnetic resonance imaging of the brain of normal neonatal foals. Vet Radiol Ultrasound 1997; 38: 102-111.

21. Hevesi A, Stanek Ch, Garamvolgyi R, Petrasi Z, Bogner P, Repa I. Comparison of the navicular region of newborn foals and adult horses by magnetic resonance imaging. J Vet Med A Physiol Pathol Clin Med 2004; 51: 143-149.

22. Schaller O. Illustrated Veterinary Anatomical Nomenclature. 2nd ed. Stuttgart, Germany: Enke Verlag; 2007.

23. Vilar JM, Arencibia A, Ramírez JA, Gil F, Latorre R, Morales I, Vazquez JM. Magnetic resonance imaging of the thorax of three dogs. Vet Rec 2003; 153: 566-568.

24. Abolmaali ND, Schmitt J, Krauss S, Bretz F, Deimling M, Jacobi V, Vogl TJ. MR imaging of lung parenchyma at $0.2 \mathrm{~T}$ : evaluation of imaging techniques, comparative study with chest radiography and interobserver analysis. Eur Radiol 2004; 14: 703-708.

25. Both M, Schultze J, Reuter M, Bewig B, Hubner R, Bobis I, Noth R, Heller M, Biederer J. Fast T1- and T2-weighted pulmonary MR-imaging in patients with bronchial carcinoma. Eur J Radiol 2005; 53: 478-488.

26. Weber U, Lambert RG, Rufibach K, Maksymowych WP, Hodler J, Zejden A, Duewell S, Kissling RO, Filipow PL, Jurik AG. Anterior chest wall inflammation by whole-body magnetic resonance imaging in patients with spondyloarthritis: lack of association between clinical and imaging findings in a crosssectional study. Arthritis Res Ther 2012; 14: R3.

27. Ley-Zaporozhan J, Ley S, Sommerburg O, Komn N, Müller FM, Schenk JP. Clinical application of MRI in children for the assessment of pulmonary diseases. RöFo 2009; 181: 419-432. 